\title{
Dimensional analysis of the human EEG and intelligence
}

\author{
Werner Lutzenberger ${ }^{a}$, Niels Birbaumer ${ }^{\text {a.b }}$, Herta Flor ${ }^{\mathrm{a}}$, Brigitte Rockstroh ${ }^{\mathrm{d}}$ and Thomas Elbert ${ }^{\mathrm{c}}$ \\ "Department of Clinical and Physiological Psychology, University of Tübingen, Tübingen (FRG), ${ }^{b}$ Dipartimento di Psicologia Generale, Universita Degli \\ Studi, Padova (Italy). "Institute of Experimental Audiology. University of Münster, Münster (FRG) and "Department of Psychology, University of \\ Konstanz, Konstanz (FRG)
}

Key words: Dimension; Intelligence; Non-linear analysis; Deterministic chaos; EEG

\begin{abstract}
The purpose of this study was the determination of the relationship between the dimensional complexity of the electroencephalogram (EEG) and the level of intelligence in humans. In two experiments 34 male subjects were divided into two groups, with high and low levels of intelligence (as measured by the intelligence quotient (IQ)). During a resting phase and various mental imagery conditions the EEG was recorded from several scalp sites. Nonlinear analysis, based on the theory of deterministic chaos, revealed that subjects with high IQs demonstrate higher dimensional complexity of the EEG attractors than subjects with low IQs only during resting conditions. During performance of the imagery tasks the less intelligent subjects increase the complexity of electrical brain dynamics such that IQ-dependency vanishes. The gross (mass) neuronal manifestation of general intelligence seems to depend on task conditions and may be related to the individual brain dynamics only when no specific task is present.
\end{abstract}

General intellectual ability as defined by the intelligence quotient (IQ) has the highest estimations of hereditability (ranging from 50 to $60 \%$ ) of all of the human behavioral attributes which have so far been analyzed in human genetic research. In contrast, specific cognitive abilities such as the speed of perception, visual memory, or social interaction are much more dependent on environmental task demands [10]. It may therefore be expected that the neuronal basis of general intelligence expresses itself particularly in situations without specific task requirements.

Previous research attempted to relate evoked potential (EP) measures of brain activity to levels of intelligence, assuming that higher intelligence should express itself in more complex waveforms [3, 6]. The failure of EP research to confirm this association may be due to the fact that during the recording of EPs subjects are asked to perform very specific, mostly visual tasks. Under these requirements, attention must be strictly focussed, and consequently, the competition between different neuronal cell assemblies involved in performing the task will be reduced $[4,6,9]$. Competition between cell assemblies may be operationally defined by the number of simultaneously active cell assemblies characterized by asynchro-

Correspondence: N. Birbaumer, Department of Clinical and Physiological Psychology, University of Tübingen, Gartenstr. 29, W-7400 Tübingen, FRG. nous (chaotic) firing patterns which subserve a particular sensory, motor, or cognitive function. Freeman [5], for example, used this term to describe the number of simultaneously bursting neuronal pools during presentation of a new, unlearnt odor in the olfactory bulb of the rabbit. During the course of conditioning the number of simultaneously active assemblies became synchronized to one single firing pattern and the behavior changed from 'search-orienting' to an orderly motor response sequence to the odor cues. This situation may be generalized to a laboratory condition with tasks that pose varying attentional demands. During concentration, inhibition in cell assemblies irrelevant for task completion and a simultaneously increased attentional resource allocation to taskrelevant brain areas may lead to a reduction of cell assembly competition [1].

One method of quantifying 'competition' among activated cell assemblies is the application of nonlinear dynamics to the spontaneous ongoing neural mass activity (as recorded through the EEG) from various cortical sites for extended periods of time during different task demands. In contrast to previous methods of EEG and EP analysis approaches derived from the theories of deterministic chaos capture unpredictable, but nonetheless regular recurrent EEG frequency patterns not detectable by traditional EEG analyses based on frequency bands in the power spectrum. Since the early eighties, mathematical tools have been developed for the analysis of dy- 
namic behavior that also take into account such nonlinear relationships. When stationary, the dynamics of any deterministic system generating a specific time series can be frozen into a static projection, known as the attractor. One measure which characterizes the attractor, and thus the dynamic behavior, is its dimensionality. Its value has been referred to as 'dimensional complexity', as structures with a higher number of dimensions are generally viewed as being more complex. Competition among oscillating neuronal cell assemblies has been shown to result in higher dimensional complexity [5] of the electroencephalogram.

It was expected that higher intelligence would result in more complex dynamics of neuronal mass activity in conditions where thoughts were allowed to float freely. Thus, subjects of higher intelligence should display EEG-patterns of higher dimensionality. The hypothesis that the dimensional complexity of the neural dynamics that generate the human EEG would increase with higher processing capacities, as measured by tests of intelligence, was examined in two consecutive studies.

In experiment I, 22 male subjects, aged between 25 and 61 years were selected from an initial subject sample of 100 to differ on a broad range of IQ levels (measured by the CFT: culture fair intelligence test of Cattell, 1972). A median split was used to divide Ss in low (IQ $<100)$ and high $(I Q \geqslant 100)$ IQ groups. A highly significant $(t=6.34$. $P<0.001$ ) difference in IQ level was achieved (Group 1: mean IQ $=84.4$, S.D. $=11.4$, range $=$ from 63 to 98 , Group 2: mean IQ $=118.2$. S.D. $=13.6$, range $=$ from 100 to 131 ).

To control for cognitive variables other than IQ, subjects were given tests of concentration and reaction time with and without distraction. The high and low IQ groups were not significantly different on any of these measures (all $t$ 's $<0.5$, all $p$ 's $>0.40$ ). Subjects were engaged in $30 \mathrm{~s}$ intervals of emotional imagery involving personal scenes of highly positive (erotic content) and negative (loss of a beloved person) valence while sitting with their eyes open. The sequence of presentation of the two emotional tasks was counterbalanced across subjects. Each imagery task was preceeded by a $30 \mathrm{~s}$ rest period. EEG was recorded from 15 sites according to the international 10-20 system (F7, F3, Fz, F4, F8, T3, C3, $\mathrm{Cz}, \mathrm{C} 4, \mathrm{~T} 4, \mathrm{~T} 5, \mathrm{P} 3, \mathrm{Pz}, \mathrm{P} 4, \mathrm{~T} 6$; impedances less than 5 $\mathrm{k} \Omega$ ). $\mathrm{Ag} / \mathrm{AgCl}$ electrodes were used for the EEG and three additional eye movement measures. All leads were referenced to the vertex electrode during recording but were changed to a linked ears reference off-line. A single ground electrode was attached to the left wrist. EEGand EOG-signals were digitized at a rate of $100 \mathrm{~Hz}$ with filter settings at 2 and $35 \mathrm{~Hz}$.

For each task a $20.48 \mathrm{~s}$ interval, composed of 2048

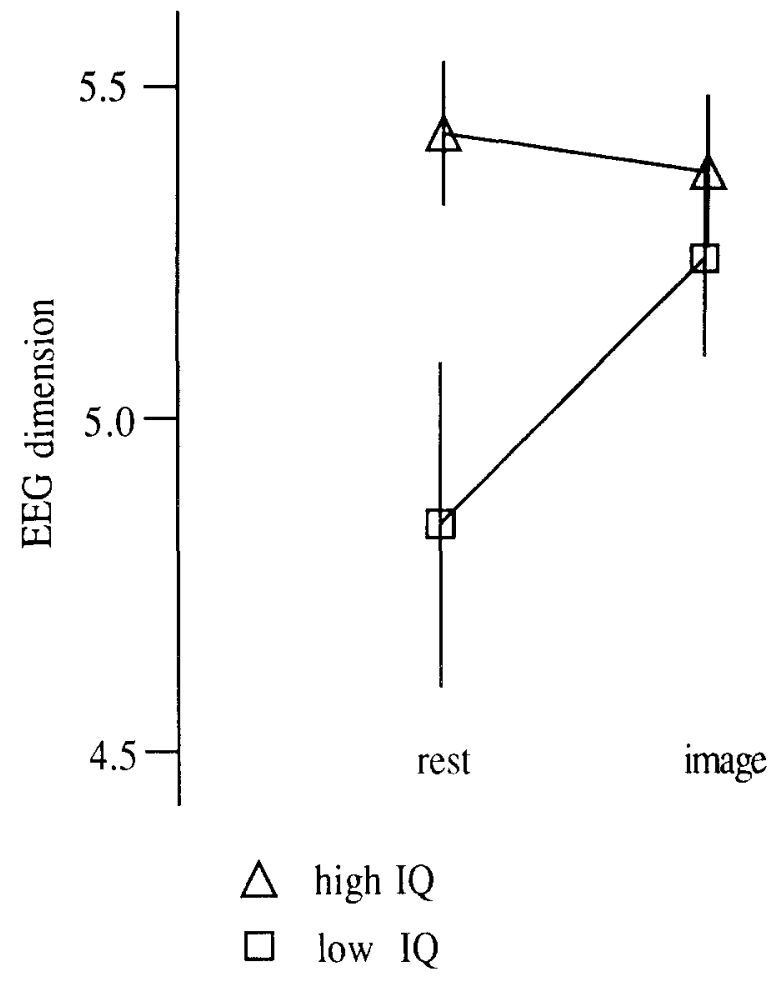

Fig. 1. Dimensional complexities (mean \pm S.D.) for different conditions, (rest. imagery) averaged across subjects with high and low IQ scores.

data points, free of eye-movements was selected for the computation. The dimensional complexity of the EEG, i.e. the fractal dimension of the reconstructed attractor of the EEG-generating system, was estimated using a singular value decomposition, which was based on the autocovariation function with time-lags ranging from 0 to 32 points. A subset of the Eigenvectors was used to reconstruct the state space. Only Eigenvectors with Eigenvalues larger than twice the smallest of all 32 Eigenvalues were selected ${ }^{1}$. Separate calculations of the dimensionality were performed for 32 equidistant points using the method of 'pointwise dimension' $[2,9,11]$. Details of the method are described in Elbert et al. [2]. The time series employed here are very small compared to the theoretical requirements for a good estimation of the dimension of the normal EEG. Although a systematic underestimation of the true values occurs. differences in the number of dimensions are reliable and may thus be interpreted $[2$, 9].

\footnotetext{
' This selection was performed in order to separate the signal from presumed noise. The criterion chosen has two advantages: It is independent of the particular gains used and it is easily reproducible among different laboratories. The alternative choice - using a fraction of the largest Eigenvalue as cut-off criterion ... would yield a high weight on slow waves, which usually determine much of the total variance.
} 
low IQ
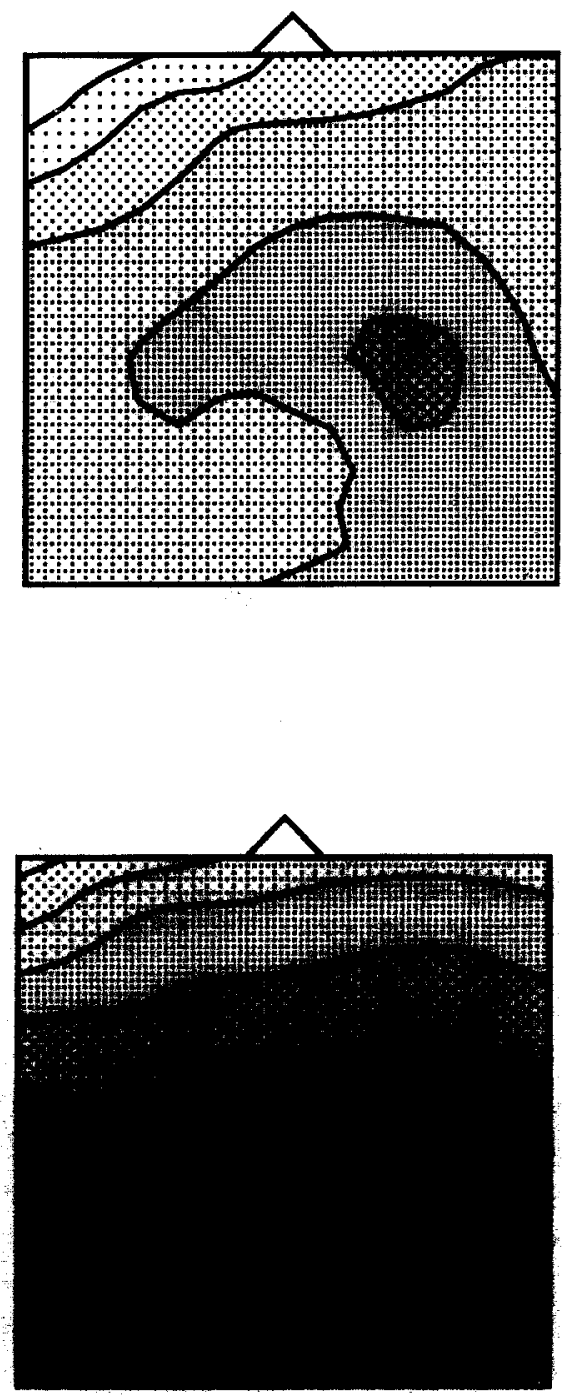

high IQ

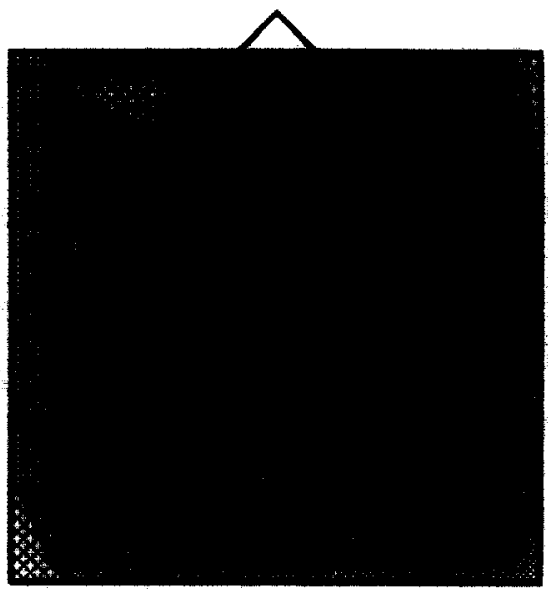

rest

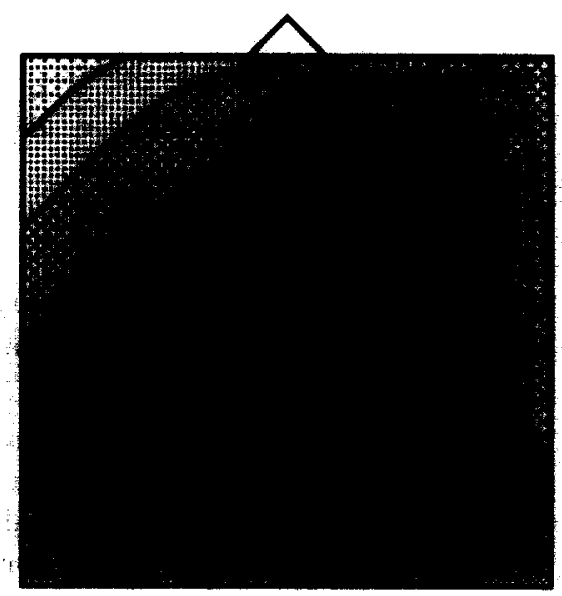

image
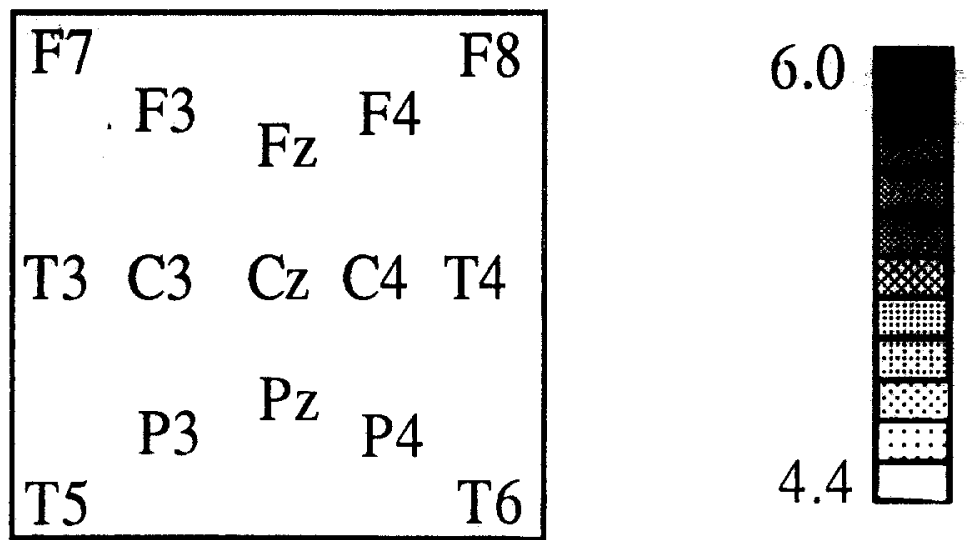

\section{EEG dimension}

Fig. 2. Maps of the dimensional complexity of the EEG averaged separately for the group with high IQ, and for the group with low IQ during rest and during imagery. For both groups, the complexity measure reveals a maximum around the right parietal region and minima at frontal sites. The maximum of the high IQ subjects is larger, particularly during resting conditions. 
A standard ANOVA with the factors group (high versus low IQ), condition (rest vs. image), and valence (positive vs. negative emotion) resulted in a clear-cut and predicted interaction of groups and conditions $\left(F_{1.20}=7.6\right.$, $P<0.012)$ and a main effect for electrodes $\left(F_{14.280}=4.4\right.$, $P<0.01)$. No interactions of the factor electrodes with tasks or groups were found. During the imagery tasks. the dimensionality of the low IQ subjects approached the values of the high IQ subjects (see Figure 1). During the resting condition, subjects with higher IQs exhibited higher dimensional complexities than subjects with low IQs $\left(t_{20}=2.2, P<0.05\right)$.

This effect originated primarily from the parietal regions: Separate tests for electrodes showed significant effects for $\mathrm{C} 3(t=2.29 . P<0.04)$. C $4(t=2.60, P<0.02)$. $\mathrm{Cz}(t=2.46, P<0.03), \mathrm{Pz}(t=2.82, P<0.01)$ and $\mathrm{P} 3$ $(t=2.20, P<0.04)$. At the other sites, no significant effects were obtained. For the $\mathrm{Pz}$ electrode, a significant correlation between the IQ-level and the dimension of the resting EEG was found $(r=0.48, P<0.02)$. Fig. 2 shows the maps of dimensional complexity averaged separately across the more and less intelligent subjects during rest and imagery conditions. Emotional content of the image had no influence on EEG complexity. In addition to the dimensional analysis, several measures based on conventional frequency analysis of the EEG (delta, theta, alpha and beta power, and the Hjorth parameters [7]) were examined. None of these measures resulted in differences between the groups.

Expt. II was performed to confirm the major findings of Expt. I and included 12 arbitrarily selected subjects. The measurement of the IQs revealed that 8 had an IQ above, and four an IQ below 100 (low IQ SS: mean $=87.25$, S.D. $=2.63 ;$ high IQ SS: mean $=115.25$. $\left.\mathrm{SD}=5.52: \quad t_{11}=9.45, \quad P<0.001\right)$. The subjects' age ranged from 19 to 40 years. Their EEG was recorded from three sites $(\mathrm{Fz}, \mathrm{Cz}, \mathrm{Pz})$ in a relaxed waking state during eyes open and eyes closed conditions ${ }^{2}$. The results of Expt. II supported the findings of Expt. I: The subjects whose IQ was above 100 all showed larger dimensional complexities (4.3-6.5) than the subjects with an IQ below 100 (3.6-4.2). This difference was again significant $\left(F_{1.10}=5.9, \quad P=0.036\right)$. Single electrode comparisons showed a significant difference only at $\mathrm{Pz}\left(t_{10}=2.99\right.$. $P<0.02)$ but not at $\mathrm{Fz}$ or $\mathrm{Cz}(P>0.15)$. Although not significant. dimensional complexity was somewhat lower in the eyes closed as opposed to the eyes open condition.

These data indicate that human general intellectual ability may be reflected in a higher complexity of the electrical brain dynamics only in situations that promote

\footnotetext{
We acknowledge the support of Drs. P. Berg and R. Cohen (Unis
} Konstanz.) in whose laboratories these data were collected. anatomically widespread interaction among different cell assemblies. The term complexity refers in this context to the reconstructed attractor dimension and a higher complexity suggests that there are more processes active that govern the major portion of neural mass dynamics as reflected in the EEG trace. Previous research has sometimes reported a relationship of level of intelligence and increased variability of electrical brain activity. If the dimensional complexity measure represents similar mechanisms then the data can be interpreted in accordance with the previous literature [4, 6]. This needs to be determined in future research. During resting periods persons with lower levels of intelligence may be characterized by a relative reduction in the variability of cortical activity with less dynamic alterations in the activity of neuronal cell assemblies, and thus lower dimensional complexities governing the EEG oscillations. Alternatively. the higher complexity observed in the more intelligent subjects during resting conditions might be related to a third unknow'n variable such as a personality trait or other cognitive abilities.

The differences in dimensionality between the high and low IQ subjects, together with the finding that the absolute values of the dimensions reached their maximum at parictal regions is in accordance with previous work showing higher complexity during sensory processing at parietal sites compared to frontal sites [8]. With increasing motor demands and during imagery the complexity also rises at frontal sites. During resting conditions subjects were asked to keep their eyes open. Thus our resting condition may represent more sensory processing than imagery or motor activation. It is conceivable that the higher dimensional complexity in the high IQ subjects during rest represents the tendency of intelligent subjects to indulge in more extended cognitive orienting and search behavior.

Whether measures of EEG complexity can be used ats tools in assessing higher brain functions must be determined by fut ure research. employing larger subject samples and a variety of cognitive tasks. Our data suggest that deterministic chatos may offer new ways of analyzing neural regulation at al gross (malss action) level.

Supported by the Deutsche Forschungsgemeinschaft (SFB 307).

1 Birbatumer. V.. Flbert. T. Canavan. A. and Rockstroh. B. Slow potentials of the cerebral cortes and behatior, Physiot. Rev. 70 (1990) $1+1$.

2 Elhert. T., Lutzenberger, W.. Rockstroh. B., Berg. P. and Cohen. R. Physical aspects of the EE(i in schiouphrencs. Biol. Psychiatry. in press.

3 Ertl. I. and Schater. F.. Brate response correlates of prechomseric intelligence. Nature, $223(1969) 421$ t22. 
4 Eysenck, H.J., The theory of intelligence and the psychophysiology of cognition. In R.J. Sternberg (Ed.), Advances in the Psychology of Human Intelligence, Vol. 3, Erlbaum, Hillsdale, NJ, 1986, pp. 1-34.

5 Freeman, W.J.. The physiology of perception. Sci. Am.. 264 (1991) 78-85.

6 Hendrickson, A.E., The biological basis of intelligence, Part I: Theory, In H.J. Eysenck (Ed.), A Model for Intelligence, Springer, Berlin, 1982, pp. 151-169

7 Hjorth, B., Time domain descriptors and their relation to a particular model for generation of EEG activity. In $G$. Dolce and $H$. Künkel (Eds.), CEAN Computerized EEG analyses, Fischer, Stuttgart, 1975, pp. 3-8.

8 Lutzenberger, W., Elbert, T., Birbaumer, N., Ray, W.J. and
Schupp, H., The scalp distribution of the fractal dimension of the EEG and its variation with mental tasks. submitted.

9 Mayer-Kress, G., Yates, F.E., Benton, L., Keidel, M., Tirsch, W. Pöppl, S.J. and Geist, K., Dimensional analysis of nonlinear oscillations in brain, heart, and muscle. Math. Biosci., 90 (1988) 155182

10 Plomin, R., Defries, J.C. and McLearn. G.E.. Behavioral Geneties, Freeman, Co., New York, 1990.

11 Ray, J.R., Wells, R., Elbert, T., Lutzenberger, W. and Birbaumer, N., Brain and chaos: dimensional estimation of sensory and hypnotic processes. In D.W. Duke and W.S. Pritchard (Eds.) Measuring Chaos in the Human Brain. World Scientific, Singapore. 1991, pp. 199-215. 